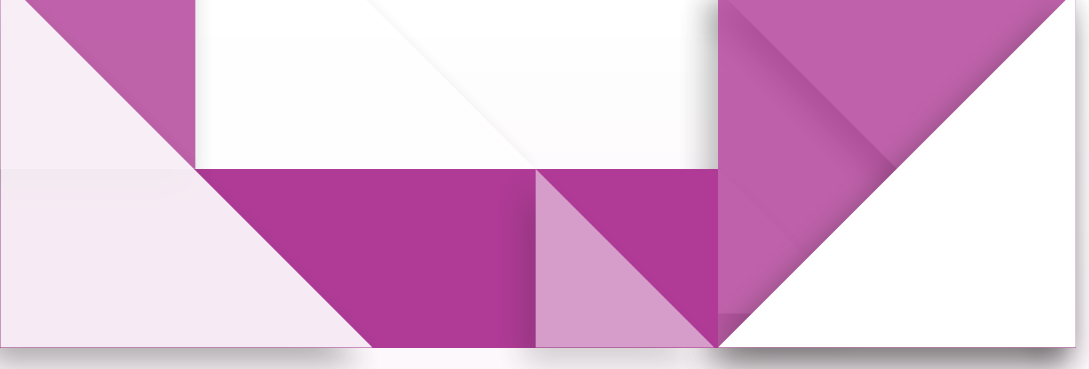

\title{
1) Saber suficiente no es suficiente: comportamientos metacognitivos al resolver problemas de demostración con el apoyo de la geometría dinámica
}

- Knowing Enough ls Not Enough: Metacognitive Behaviors When Solving Proof Problems with the Support of Dynamic Geometry

- Conhecer o suficiente não é suficiente: comportamentos metacognitivos ao resolver problemas de demonstração com o suporte da geometria dinâmica

Magíster en Tecnologías de la Información aplicadas a la Educación. Profesor de Matemáticas. Universidad Pedagógica Nacional. Departamento de Matemáticas. Bogotá, Colombia. ¡csuaf@pedagogica.edu.co orcid.org/0000-0002-5700-4999

\section{Resumen}

Los problemas de demostración demandan poner en juego distintos conocimientos y habilidades instrumentales cuando se cuenta con apoyo de la geometría dinámica. Sin embargo, como se muestra en este documento, el conjunto de conocimientos de un individuo y su grado de instrumentalización del software no son los únicos aspectos relevantes en el proceso de resolución o en la naturaleza de la respuesta obtenida. Apoyados en dos grupos de estudiantes para profesor de matemáticas con un nivel de formación matemática distinta, mostramos qué aspectos metacognitivos como el control, la regulación y la evaluación de las acciones ejecutadas se convierten en elementos que pueden llevar a un grupo, con un conocimiento matemático reducido, a obtener mejores resultados que un grupo con un conocimiento profundo de la disciplina. Mostramos cómo el trabajo grupal y el uso de la geometría dinámica inciden positivamente en el proceso de resolución y favorecen aspectos de orden metacognitivo.

Palabras clave

resolución de problemas; geometría dinámica; metacognición; problemas de demostración 


\section{Abstract}

Proof problems demand to put into play different knowledge and instrumental skills when it is supported by dynamic geometry. However, as we show in this paper, an individual's set of knowledge and the degree of instrumentalization of the software are not the only relevant aspects in the resolution process or in the nature of the response obtained. Supported in two groups of mathematics preservice teacher with a different level of mathematical training, we show that metacognitive aspects such as control, regulation and evaluation of executed actions become elements that can lead to a group, with mathematical knowledge reduced, to obtain better results than a group with a deep knowledge of the discipline. We show how group work and the use of dynamic geometry have a positive effect on the resolution process and promote metacognitive aspects.

Keywords

problem solving; dynamic geometry; metacognition; proof problems

\section{Resumo}

Os problemas de demonstração exigem colocar em jogo diferentes conhecimentos e habilidades instrumentais quando são suportados pela geometria dinâmica. No entanto, conforme o conteúdo deste documento, o conjunto de conhecimentos de um indivíduo e o grau de instrumentalização do software não são os únicos aspectos relevantes no processo de resolução ou na natureza da resposta obtida. Apoiados em dois grupos de alunos, futuros professores de matemática, com um nível diferente de treinamento matemático, evidenciamos que aspectos metacognitivos como o controle, a regulação e a avaliação de ações executadas tornam-se elementos que podem levar a um grupo, com conhecimento matemático reduzido, a obter melhores resultados do que um grupo com um profundo conhecimento da disciplina. Mostramos como o trabalho em grupo e o uso da geometria dinâmica têm um efeito positivo no processo de resolução e favorecem aspectos metacognitivos.

Palavras-chave

resolução de problemas; geometria dinâmica; metacognição; problemas de demonstração 


\section{Introducción}

En la enseñanza y aprendizaje de la geometría se reconoce el uso y relevancia de los programas de geometría dinámica (PGD O $G D)$. La naturaleza de este recurso promueve el descubrimiento de relaciones de dependencia que difícilmente se podrían observar en configuraciones estáticas. Esto ha despertado nuevos intereses en la enseñanza de la geometría, entre los que se encuentra la demostración (Hanna, 2000, p. 12). En esta vía se reconocen investigaciones que estudian la comprensión de los estudiantes sobre la demostración y formas para apoyar dicha comprensión, algunas de estas apoyadas en la GD (Hanna, 2000, p. 13; Marrades y Gutiérrez, 2000). Dada la dificultad de los estudiantes al afrontar la demostración, algunos estudios han involucrado constructos teóricos como la resolución de problemas (Furinghetti y Morselli, 2009; Koichu y Leron, 2015).

La investigación sobre la resolución de problemas ha incorporado elementos como la metacognición, pues se reconoce una problemática en los estudiantes al afrontar este tipo de tareas (Erbas y Okur, 2012; Furinghetti y Morselli, 2009)problem solving episodes, and metacognitions of five Turkish high school freshmen and explore the interplay of these on their problem solving success in mathematics. Participants worked on ten problems selected among the released mathematical literacy items used in Programme for International Student Assessment (PISA. La metacognición es importante en la resolución de problemas al permitir manejar la complejidad del problema mismo y evaluar el progreso hacia los logros. Dentro de los resultados más relevantes obtenidos al respecto se encuentra el hecho de que (1) individuos con un conocimiento básico pueden obtener mejores resultados al resolver problemas que individuos con un conocimiento superior, cuando los primeros cuentan con habilidades metacognitivas superiores (Cai, 1994) y (2) que el proceso de resolución de problemas se favorece gracias a la metacognición cuando se consideran configuraciones colaborativas y la presencia de recursos externos (Kim, Park, Moore y Varma, 2013, p. 381).

Abordar la demostración de enunciados geométricos no solo involucra contar con un vasto conjunto de conocimientos geométricos, también demanda habilidades metacognitivas que permitan controlar y regular los conocimientos de los que se dispone, proyectar rutas de trabajo adecuadas y descartar estrategias no afortunadas para alcanzar la demostración de algún enunciado. Aun cuando se cuenta con investigaciones sobre la resolución de problemas y la metacognición, donde interviene la $G D$, el estudio en el marco de la demostración en geometría no ha sido contemplado. En este documento presentamos un estudio de los comportamientos metacognitivos (CM) y el proceso de resolución, llevado a cabo por dos grupos con un nivel de formación matemática distinta, al afrontar problemas de demostración geométricos con el apoyo de GD. El objetivo de este estudio es identificar y comparar la naturaleza de este proceso y determinar la forma en que sus conocimientos inciden en él.

\section{Marco teórico}

\section{Problemas de demostración}

Para Nunokawa (2010), un problema es una situación que no se relaciona inmediatamente con un conocimiento o procedimiento matemático que juegue un rol en la solución. Esto 
lleva a analizar la situación abordada y a usar heurísticas que permitan trazar una ruta de solución, que pueden ser específicas o generales a un dominio de conocimiento y promueven una estrategia general de resolución o apenas un pequeño avance.

Establecer una demostración puede considerarse un problema, pues se reconocen condiciones iniciales y un resultado a obtener, en el que se transita gracias al uso de elementos de un sistema teórico de referencia a través de un razonamiento deductivo (Perry, Samper, Camargo y Molina, 2013). Denominaremos a estas situaciones problemas de demostración, conservando así la idea propuesta por Polya al referirse a estos como una clase de problemas en que los estudiantes proveen una justificación a alguna aserción, explícita en el enunciado del problema o descubierta y formulada como parte de la tarea (Marrades y Gutiérrez, 2000).

\section{Episodios de la resolución de problemas}

Kuzle (2015) propone un modelo que caracteriza el proceso de resolución de problemas cuando media la GD. Dicho modelo involucra siete episodios (figura 1), los cuales son presentados por Schoenfeld originalmente e interpretados y complementados por Kuzle como fundamento teórico de su investigación. Aun cuando el modelo sugiere una trayectoria lineal entre los episodios, el recorrido por estos puede darse de forma cíclica, incluso omitiendo algunos episodios. Una descripción de estos se presenta a continuación.

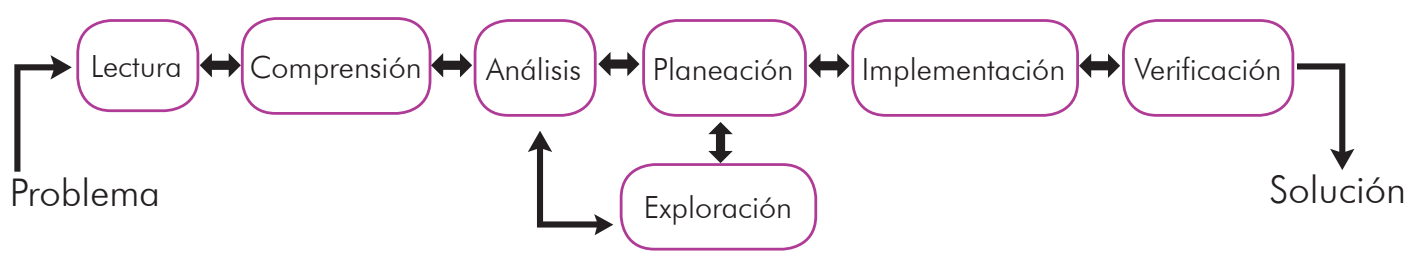

Figura 1. Episodios de la resolución de problemas

Fuente: elaboración propia a partir de Kuzle, 2015. 
Tabla 1. Descripción de episodios resolución de problemas

\begin{tabular}{|c|c|}
\hline Episodio & Descripción \\
\hline Lectura (L) & $\begin{array}{l}\text { Tiene lugar cuando un sujeto lee el enunciado del problema. Esto incluye el reconocimiento de } \\
\text { las condiciones del problema y sus partes. }\end{array}$ \\
\hline Comprensión (C) & $\begin{array}{l}\text { Engloba los intentos de un sujeto por clarificar el significado del problema, sus partes y la } \\
\text { relación entre estas. Se identifican objetivos del problema, este se representa y se evalúa el } \\
\text { conocimiento personal respecto al demandado. }\end{array}$ \\
\hline Análisis (A) & $\begin{array}{l}\text { Cuando no hay una forma aparente de proceder después de haber leído el problema, tiene } \\
\text { presencia un ejercicio de análisis. El estudiante descompone el problema en otros, examina } \\
\text { relaciones entre los datos, las condiciones y el objetivo del problema, seleccionando posibles } \\
\text { rutas de solución. }\end{array}$ \\
\hline Exploración (E) & $\begin{array}{l}\text { Se realiza un recorrido por el espacio del problema, descubriendo información que pueda ser } \\
\text { incorporada en episodios de análisis, planeación o implementación. Aquí tienen presencia } \\
\text { distintas heurísticas, la examinación de problemas relacionados, el uso de analogías, etc. La } \\
\text { distinción entre la exploración y el análisis puede darse en su estructura. Mientras el análisis se } \\
\text { caracteriza por ser bien estructurado, ciñéndose a las condiciones o logros del problema, la } \\
\text { exploración es menos estructurada, desligada del problema original. }\end{array}$ \\
\hline Planeación (P) & $\begin{array}{l}\text { El sujeto establece cómo proceder para resolver el problema. Algunas rutas pueden ser trazadas } \\
\text { y consideraciones o anticipaciones sobre estas rutas se pueden vislumbrar, lo que puede llevar o } \\
\text { no a su implementación. }\end{array}$ \\
\hline Implementación (I) & $\begin{array}{l}\text { Se ejecutan acciones para operar con la información de la que se dispone. Estas acciones } \\
\text { pueden apoyarse en planes considerados. Sin embargo, se puede implementar alguna } \\
\text { estrategia sin que esta se planificara. }\end{array}$ \\
\hline Verificación (V) & $\begin{array}{l}\text { Se revisan los resultados obtenidos y su correspondencia con lo solicitado en el enunciado del } \\
\text { problema. Este episodio no es punto final del proceso de resolución, dado que puede llevar } \\
\text { al sujeto al punto de partida nuevamente si el resultado obtenido no es consecuente con lo } \\
\text { solicitado. }\end{array}$ \\
\hline
\end{tabular}

Fuente: elaboración propia.

\section{Metacognición}

La metacognición se origina en la década de los setenta, pero apenas en la década de los ochenta la investigación en educación matemática puso atención a este asunto y lo incorporó en algunos desarrollos teóricos dada la posibilidad de analizar con éste el desempeño en tareas matemáticas (Schneider y Artelt, 2010). En la literatura se reconoce una alta presencia de la definición propuesta por Flavell (1979, citado por Karsli, 2015, p. 36) y en esta dos aspectos generales: un dominio cognitivo y una función de monitoreo. Para Flavell la metacognición se concibe como:

1. El conocimiento personal sobre los procesos cognitivos propios y los productos, o cualquier otro aspecto, relacionado a estos; así como,
2. el monitoreo activo y la regulación y orquestación consecuente de esos procesos en relación a los objetos cognitivos en los cuales ellos yacen, por lo general en función de un logro u objetivo concreto.

Esta definición acude al conocimiento de los procesos de pensamiento personales y la habilidad de controlar, monitorear y regular los comportamientos de aprendizaje personales bajo la intención de alcanzar una comprensión profunda y una resolución de problemas efectiva (Lin y Sullivan, 2008). La metacognición guarda relación con estrategias como la planeación, monitoreo y evaluación del desempeño. Estas estrategias apoyan el pensamiento de los estudiantes en la solución de un problema, verificando su progreso de aprendizaje, planeando y ejecutando 
cambios en las actividades cognitivas en curso, dados los resultados que se obtengan, y monitoreando y comparando resultados cognitivos con criterios externos e internos (Karsli, 2015).

La influencia de las relaciones sociales en el aprendizaje permiten reconocer nexos entre la metacognición y la interacción social (Lin y Sullivan, 2008), lo que ha llevado este elemento más allá de la mirada individual dada en su génesis. La metacognición puede verse desde una perspectiva social que involucra el monitoreo y control del conocimiento de uno mismo y de otros (Chiu, Jones, y Jones, 2013). En esta dimensión social se reconocen los efectos del trabajo colaborativo, escenario donde la resolución de problemas promueve que el monitoreo y control de un sujeto sobre el comportamiento de sus compañeros apoye una resolución exitosa (Chiu et al., 2013). La literatura reporta que concebir la metacognición como socialmente compartida conlleva a un mejor desempeño dentro de los grupos. Sin embargo, la investigación en esta línea se mantiene en un nivel básico (Raes, Schellens, De Wever y Benoit, 2016). Dentro de las fuentes externas metacognitivas se considera también que el ambiente donde esta interacción toma lugar promueve la metacognición. Esta metacognición promovida por el ambiente de interacción apoya la metacognición individual y la social. Esto es, realimenta y modifica las concepciones de un sujeto o de un grupo y sirve como referente o recurso de validación de las ideas formuladas. En nuestro caso, la GD es ejemplo de estas fuentes externas.

\section{Metodología}

En este documento analizamos el trabajo de dos parejas de estudiantes al resolver cuatro problemas de demostración con ayuda de GD. Exploramos y comprendemos las dinámicas que tienen presencia en esta configuración dentro de un contexto particular, como lo es la resolución de problemas. Por ello, la investigación hace parte de un estudio de caso cuya unidad de análisis es el proceso de resolución de los problemas (Baxter y Jack, 2008).

\section{Contexto del estudio}

Dos estudiantes para profesores de primer semestre (Ana y Juan) y dos de cuarto semestre (Caro y Paul), de Licenciatura en Matemáticas de la Universidad Pedagógica Nacional (Bogotá, Colombia) fueron involucrados. La elección de trayectorias académicas distintas atendía al deseo de comparar el proceso de resolución y los comportamientos metacognitivos exhibidos en cada grupo con la profundidad en su formación matemática. Ana y Juan apenas habían tomado un curso en que se realizan aproximaciones a definiciones y propiedades de objetos geométricos (rectas, circunferencias, cuadriláteros y triángulos), se promueven procesos de argumentación y se usa de manera inicial la GD, mientras que Caro y Paul habían tomado cuatro cursos de geometría, estudiando 
formalmente objetos y relaciones en el plano y el espacio, desarrollando una habilidad en el manejo de la GD.

Para ambos grupos era común la metodología de los cursos. Esta es la propuesta metodológica formulada por el grupo de investigación $A E \bullet G$ (Perry et al., 2013), a través de la cual los elementos teóricos estudiados en clase surgen como resultado de un trabajo grupal en el que los estudiantes se involucran en la resolución de problemas y la GD es protagonista, al permitir que procesos de conjetura y justificación tengan lugar. Las producciones de los estudiantes se someten a la evaluación de sus compañeros, quienes las apoyan o refutan. Los elementos aceptados conforman un sistema teórico (definiciones, teoremas y postulados) en el marco de la geometría euclidiana.

\section{Diseño de la secuencia}

Se contemplaron problemas de demostración que involucraran asuntos del primer curso de geometría. Frente al recurso tecnológico, los problemas situaban a los estudiantes en un escenario en el que era indispensable el uso de la GD para operar con los datos dados. Los problemas se diferenciaban por los objetos geométricos involucrados, las relaciones geométricas estudiadas y el uso dado a la GD en cada uno.

\section{Acopio de datos}

Se conformaron parejas de estudiantes del mismo semestre. En el transcurso de una semana se reunió el investigador (autor del documento), con cada pareja para resolver cada problema. Cada pareja contó con un computador con GeoGebra (dada la familiaridad de los estudiantes con el programa). El investigador acompañó a los grupos sin participar en la discusión que se generó entre sus miembros. Él les solicitaba a los estudian- tes verbalizar sus pensamientos cuando se presentaban pausas o silencios prolongados con el fin de registrarlos.

Se utilizó Camtasia para registrar la interacción en la pantalla del computador, las conversaciones sostenidas entre los estudiantes y una captura, con ayuda de la cámara frontal del computador, de sus manifestaciones corporales; las producciones escritas de los estudiantes al abordar cada problema también se acopiaron. Al finalizar el trabajo con cada grupo el investigador realizaba algunas preguntas para obtener información sobre episodios que requirieran una explicación adicional.

Los audios de las grabaciones fueron transcritos y fragmentados teniendo en cuenta los objetivos de los estudiantes durante el proceso de resolución. Esto permitió convertir cada transcripción en una secuencia de bloques. Los registros generados por cada grupo fueron analizados de manera independiente para reconocer episodios de resolución, su duración y los CM exhibidos en ellos. Posteriormente, se realizó un cuadro comparativo del trabajo realizado por los grupos, analizando la presencia e incidencia de la metacognición en el proceso de resolución y estudiando la forma en que la GD, como recurso externo, se involucró dentro del proceso.

\section{Un episodio emergente}

El modelo de Kuzle no permitió codificar todas las acciones realizadas por los estudiantes. Se reconocieron momentos del proceso de resolución en que ellos establecían resultados parciales o recogían resultados del trabajo realizado. Esto llevó a considerar un nuevo episodio que se describe a continuación. 
Tabla 2. El episodio de síntesis

Se recogen resultados obtenidos al realizar distintos esfuerzos para resolver el

Síntesis problema. Estos resultados, que pueden ser parciales, provienen de la exploración o análisis realizado en una situación particular, o las creencias de quien afronta el problema.

Fuente: elaboración propia.

\section{Un ejemplo de lo ocurrido}

Por motivos de extensión, presentamos el trabajo realizado por Caro y Paul alrededor de uno de los problemas propuestos, el cual se muestra a continuación. Esta elección atiende a la naturaleza inductiva de la estrategia de solución y la forma rígida en que la GD se involucra. Se utilizan las iniciales de cada episodio de resolución entre paréntesis cuadrado para señalar su presencia.

Construya un cuadrilátero que cumpla la siguiente propiedad: las bisectrices de dos ángulos adyacentes determinan un ángulo recto. Formule conjeturas y justifíquelas.

\section{Las bisectrices del cuadrilátero}

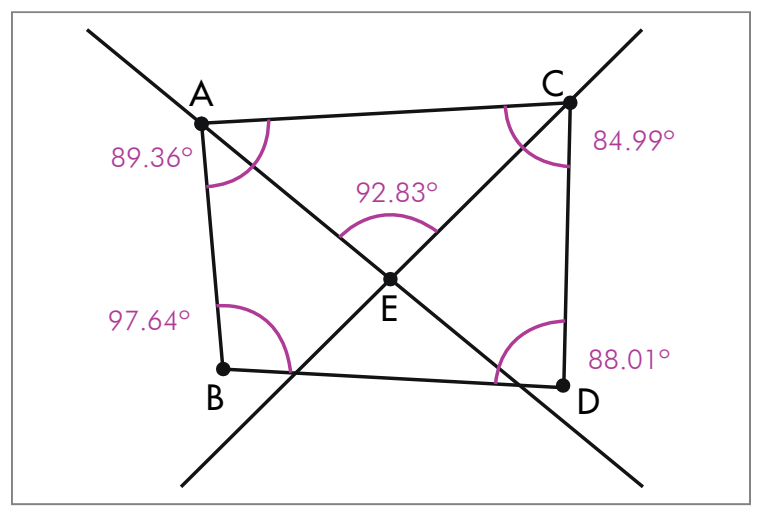

Figura 2. Cuadrilátero sin alguna propiedad

Fuente: elaboración propia.

Caro inicia realizando una lectura del enunciado sin hacer énfasis o repetir alguna parte de este [L]. Al finalizar, Paul sugiere involucrar GeoGebra y construir un cuadrilátero $[P]$, preguntando a Caro sobre la pertinencia de esta idea, a lo que Caro manifiesta aceptación. Paul representa gráficamente el cuadrilátero [l] y Caro le pide que construya las bisectrices de los ángulos involucrados (figura 2). Caro determina las medidas de los ángulos a los que se les construyó su bisectriz y propone manipular [P] el cuadrilátero con el fin de identificar las propiedades de este, en función de sus ángulos, que hacen que la perpendicularidad de las bisectrices se satisfaga. Para Paul no es claro qué ángulos deben ser rectos, 
por lo que Caro le comenta que es el ángulo determinado por las bisectrices el que debe medir 90 [C]. Caro determina las medidas de los otros ángulos del cuadrilátero y la del ángulo determinado por las bisectrices (ángulo E) [I] a la vez que hace explícito que, con base en estos, determinarán el tipo de cuadrilátero que cumple la propiedad.

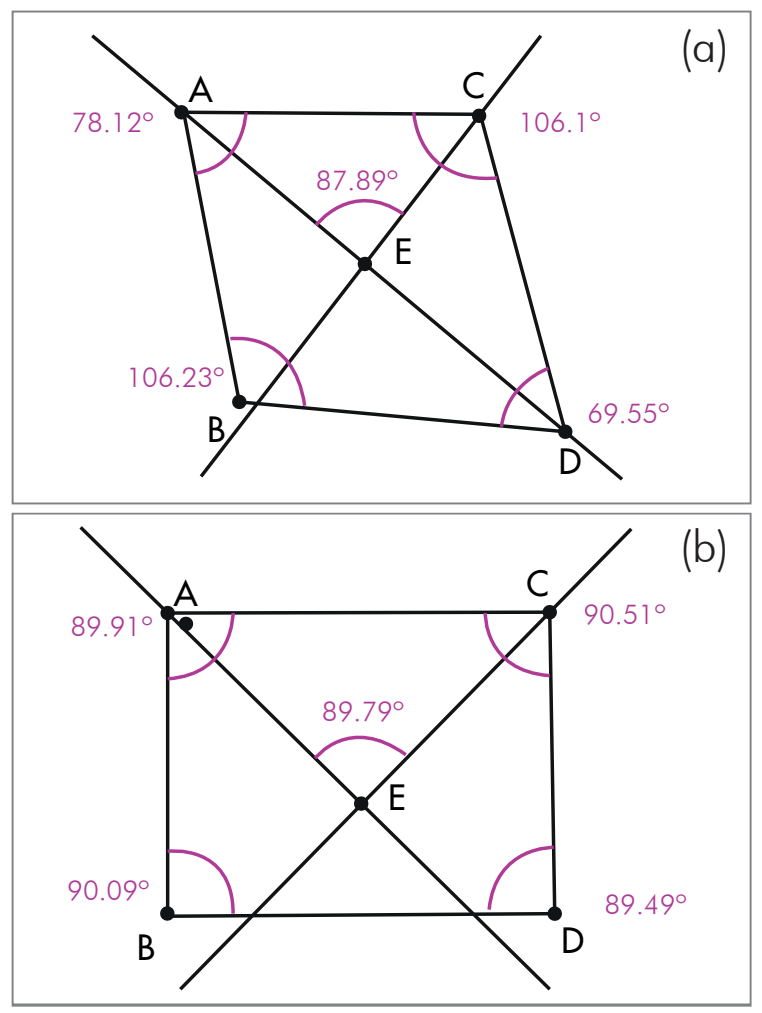

Figura 3. Ángulos opuestos congruentes (a) y ángulos adyacentes rectos (b)

Fuente: elaboración propia.

Paul le pide a Caro que arrastre los puntos y al hacer esto [E] establecen un primer resultado: no cualquier cuadrilátero satisface esta condición. Este resultado hace que Paul considere que una propiedad del cuadrilátero que hace que la perpendicularidad de las bisectrices se cumpla es que dos de sus ángulos opuestos sean congruentes. Apoyados en las medidas de los ángulos, arrastran los puntos B y $C$ hasta que sus medidas son aproximadamente iguales, pero la representación gráfica en pantalla muestra que el ángulo $\mathrm{E}$ no es recto (figura $3 a$ ), lo que lleva a Paul a descartar esta posibilidad. Ahora Paul propone $[\mathrm{P}]$ que se realice una exploración en cuadriláteros conocidos por ellos.

Caro no considera la propuesta de Paul y, apoyada en la representación en pantalla, inicia un análisis $[A]$ en el que involucra algunos elementos teóricos con el fin de justificar que los ángulos $\mathrm{A}$ y $\mathrm{C}$ deben ser rectos. Esta propuesta no es compartida por Paul, pero Caro propone [P] que se evalúe dicha posibilidad modificando el cuadrilátero. Paul arrastra el punto $B$ [I] pero las medidas de los ángulos no dan exactamente 90 (figura 3b). Aun así, la aproximación de las medidas permite ver que el ángulo E tiene una medida cercana a 90. Esto lleva a Paul a pensar que el rectángulo cumple con lo solicitado.

La sospecha de Paul lleva a que el grupo analice lo que ocurre en cuadriláteros particulares [P]. Caro propone que se transforme el cuadrilátero construido en un rectángulo. Al arrastrar los vértices del cuadrilátero [I] para lograr esto se observa que el ángulo $\mathrm{E}$ tiene un valor muy cercano a 90 (figura 4a). Paul sugiere realizar una construcción robusta del cuadrilátero para verificar este resultado [V], pero Caro lo interrumpe y asegura, basada en la construcción en pantalla, que saber que las medidas de los ángulos $A$ y $C$ es 90 , conlleva a que el ángulo $\mathrm{E}$ mide 90 . Al final ambos estudiantes, apoyados en este resultado, mencionan [S] que la propiedad solicitada en el problema se cumple cuando el cuadrilátero es cuadrado o rectángulo, explicando que el cumplimiento de la propiedad en el cuadrado se da porque este es rectángulo también. 


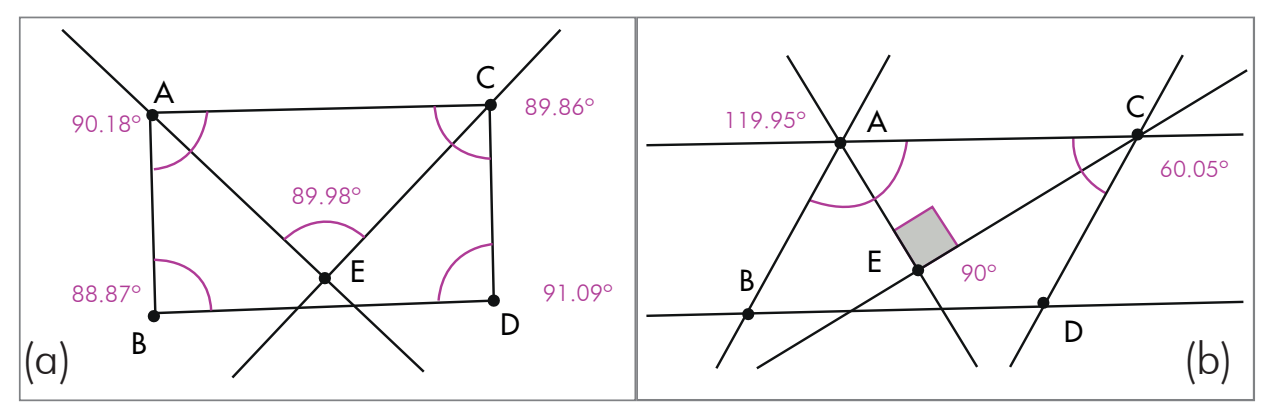

Figura 4. Una aproximación al rectángulo (a) y paralelogramo (b)

Fuente: elaboración propia.

Paul sugiere ahora considerar un paralelogramo [P], para lo cual realiza una construcción, apoyado en rectas paralelas [I], así como las bisectrices de dos ángulos adyacentes en él (figura 4b). Para Paul la propiedad parece cumplirse y al hallar la medida del ángulo determinado por las bisectrices, esta da exactamente 90, resultado que lo lleva a señalar que en cualquier paralelogramo [S] esta propiedad es verdadera. Apoyándose en este resultado, Caro señala que el rectángulo y el cuadrado cumplen esta propiedad, pues ambos son paralelogramos. Paul arrastra uno de los vértices del paralelogramo en la pantalla [V], con lo que observan que bajo arrastre esta propiedad se conserva.

Paul propone $[\mathrm{P}]$ construir un trapecio. Caro no sabe si construir un trapecio ordinario o uno isósceles, a lo que Paul responde que un trapecio ordinario. Caro construye dos rectas paralelas [I] y dos segmentos de forma tal que en pantalla se puede observar un trapecio (figura 5a). Después de ello, Paul construye las bisectrices de los ángulos que comparten uno de los lados paralelos y al hallar la medida del ángulo determinado por estos rayos, observan que esta no da 90, resultado que los lleva establecer que el trapecio ordinario no cumple la propiedad y a analizar qué ocurre en el trapecio isósceles [P], para lo cual arrastran los extremos de los segmentos de los lados no paralelos [I], buscando que sus longitudes coincidan. El resultado en pantalla les muestra que bajo esta configuración el ángulo determinado por las bisectrices no es recto (figura 5b). Por lo anterior, Paul y Caro descartan este cuadrilátero como solución [S]. 

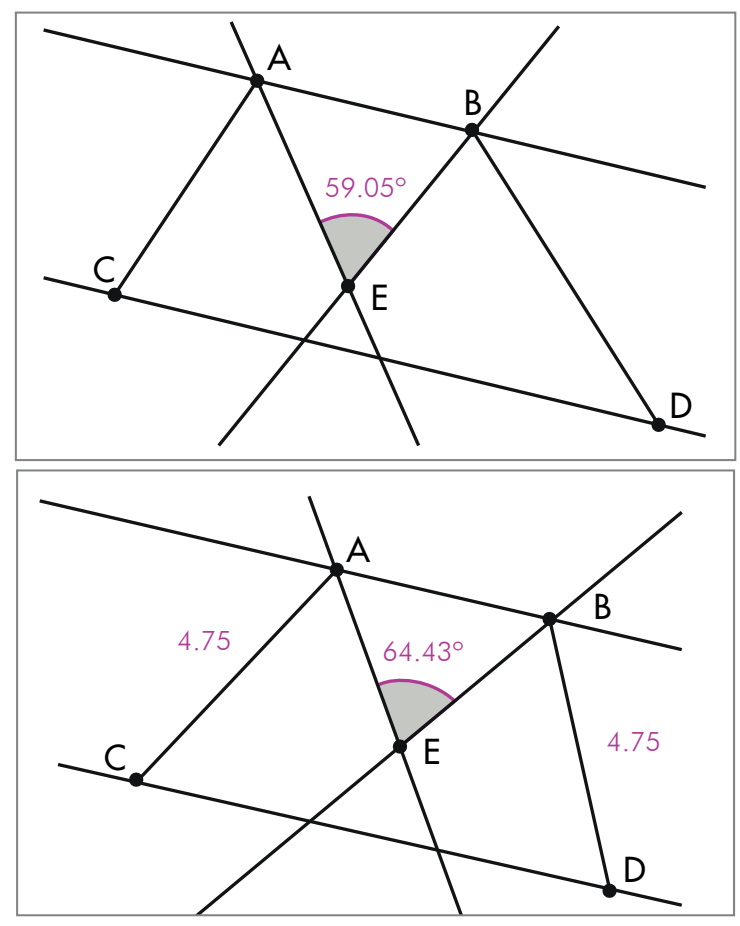

Figura 5. Estudiando los trapecios

Fuente: elaboración propia.

Paul sugiere ahora ensayar con una cometa $[\mathrm{P}]$. Al inicio dudan sobre las condiciones de este cuadrilátero, pero recuerdan que en este se cumple la congruencia de dos lados adyacentes. Antes de construir este cuadrilátero, Paul propone construir un rombo y evaluar el cumplimiento de la propiedad allí, idea rechazada por Caro en cuanto este cuadrilátero es un paralelogramo. Retomando la construcción de la cometa [l], ambos estudiantes tienen problemas al no saber cómo involucrar las herramientas de GeoGebra. Su estado de incertidumbre los lleva a construir un cuadrilátero en el que apenas dos lados adyacentes son congruentes y los otros tienen longitudes cercanas (figura 6). Ellos construyen las bisectrices y apoyados en la representación gráfica establecen el no cumplimiento de la propiedad. Caro propone determinar la medida del ángulo, arrastrar uno de los vértices de tal forma que los lados no congruentes lo sean y corroborar su apreciación. Cuando Paul hace esto, corroboran que el ángulo determinado por las bisectrices no es recto, lo que los llevó a descartar este cuadrilátero y establecer, con base en lo previamente realizado, que la propiedad estudiada solo tiene presencia en los paralelogramos [S]. Paul le pregunta a Caro si algún cuadrilátero falta por ser evaluado [V], a lo que ella responde que ya se han evaluado los cuadriláteros conocidos y que el problema ha sido resuelto.

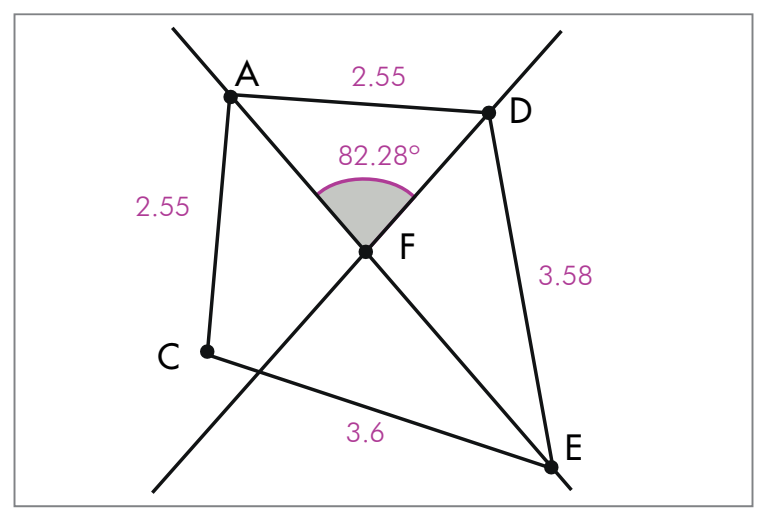

Figura 6. Construcción de la cometa

Fuente: elaboración propia.

El investigador les indica que revisen el enunciado del problema con el objetivo de no dejar de lado algún requerimiento, pues ellos consideraban que no había algo más por hacer. Los estudiantes realizan esto [L], identificando la solicitud de formular conjeturas y justificarlas. Ellos retoman la construcción del paralelogramo en GeoGebra y empiezan a mencionar algunos hechos geométricos que en este tipo de cuadrilátero se satisfacen [A] como lo son la congruencia de lados y ángulos opuestos y que las medidas de los ángulos adyacentes suman 180 . 
Tabla 3. Caracterización de episodios

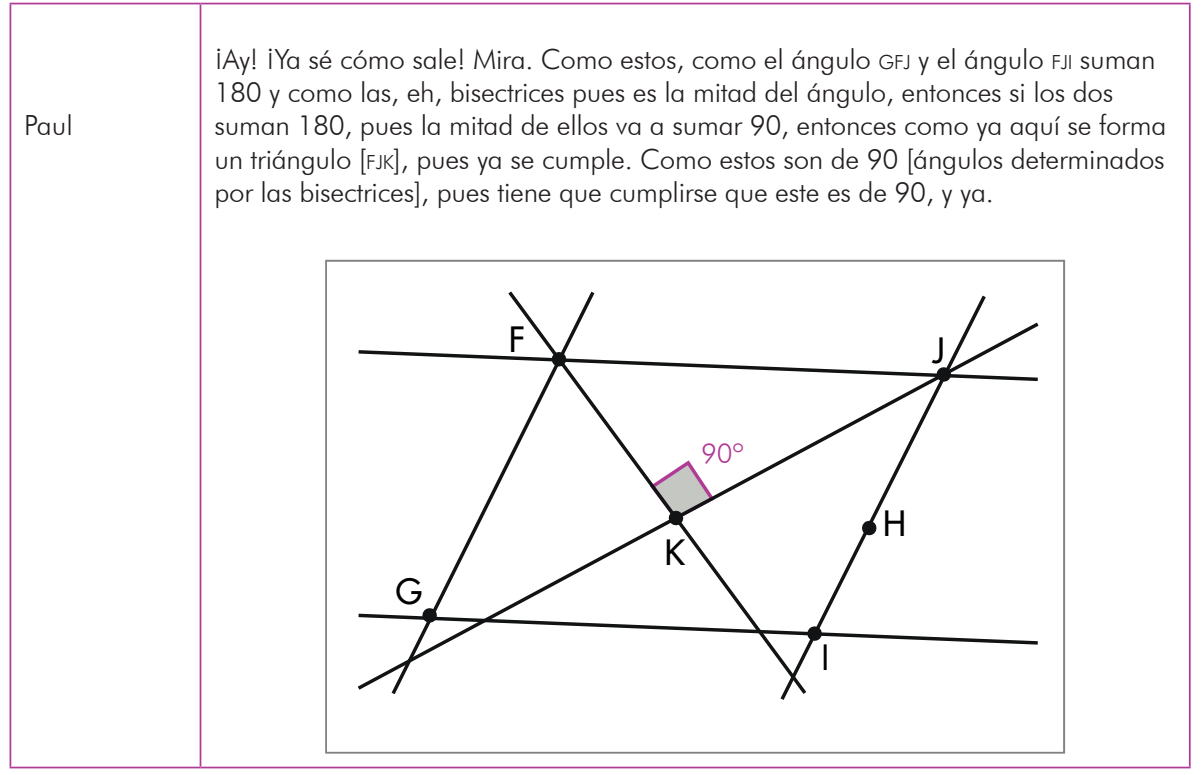

Fuente: elaboración propia.

En la intervención repentina de Paul (tabla 3) se parte de que la suma de las medidas de los ángulos GFJ y FJ es 180. Él luego involucra las bisectrices de estos ángulos para asegurar que la suma de las medidas de los ángulos KFJ y KJF tiene que dar 90. Haciendo uso del triángulo FJK, junto a lo que se acaba de establecer y un hecho geométrico que señala que en el triángulo la suma de las medidas de sus ángulos da 180, Paul asegura que el ángulo FKJ debe ser recto. Caro retoma las ideas de Paul y repite los pasos claves de la justificación propuesta por él [V]. Paul regresa al enunciado del problema y menciona que la conjetura del trabajo realizado [S] es que en los paralelogramos la propiedad involucrada se cumple.

\section{Análisis de Caro y Paul}

\section{Descripción del proceso de resolución}

Paul y Caro se comprometieron principalmente con acciones de planeación e implementación, dada la frecuencia de estos episodios (tabla 4), mientras que acciones de comprensión y exploración fueron casi nulas. Un poco más del $40 \%$ del tiempo empleado en el proceso de resolución se destinó a realizar acciones de implementación, mientras que acciones dirigidas a la comprensión del problema emplearon un tiempo reducido. 
Tabla 4. Caracterización de episodios

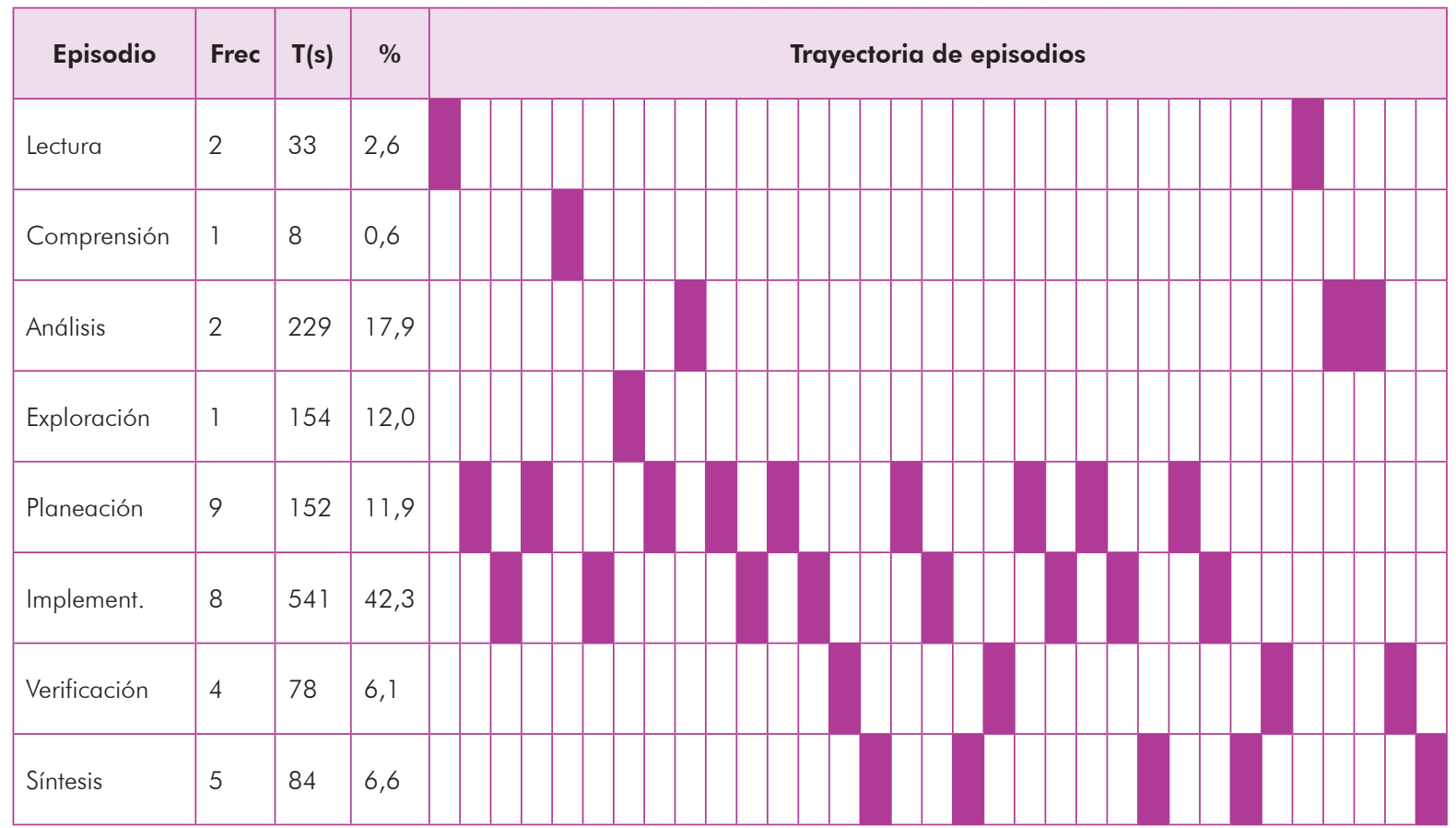

Fuente: elaboración propia.

El proceso de resolución (tabla 4) permite reconocer un momento inicial de lectura, seguido de un ejercicio de planeación e implementación que llevó a proponer rutas de trabajo. A partir de este momento, el trabajo se tornó cíclico pues se analizó lo que ocurría en cuadriláteros particulares. Esto involucró planear e implementar la construcción del cuadrilátero y a descartar o validar hipótesis. Al finalizar este trabajo reconocieron una propiedad entre ellos y procedieron a justificarla. La justificación no requirió mucho tiempo, lo que permite asegurar que para ellos la dificultad del problema se concentró principalmente en el proceso de conjetura. Al final del trabajo se verificó la justificación realizada y formularon la conjetura que sintetizaba el trabajo realizado. Sin embargo, esta conjetura reportó apenas el cumplimiento de la propiedad en un cuadrilátero particular (paralelogramo) y dejó de lado la posibilidad de reconocer una propiedad general que involucraba otros cuadriláteros.

\section{Comportamientos metacognitivos evidenciados}

El trabajo de este grupo exhibió los CM presentados a continuación. En el episodio de lectura se reconocieron comportamientos relacionados con la identificación de partes del problema que pudieran ser omitidas y la lectura de las partes principales del enunciado, acciones que permitieron comprender lo que debía realizarse y dirigir sus acciones hacia la justificación de la propiedad descubierta. En el episodio de comprensión comportamientos como retomar las condiciones expuestas en el enunciado del problema permitieron orientar las acciones que se debían realizar. El episodio de análisis deja ver comportamientos ligados a la justificación como lo fue la extracción de información de representaciones gráficas, el uso de hechos geométricos para soportar afirmaciones y el establecimiento de conclusiones soportadas en la exploración realizada en GD. 
En el episodio de exploración se observaron comportamientos como la manipulación de objetos geométricos y el examen de relaciones entre ellos, la anticipación de resultados, el uso de representaciones gráficas en la formulación de afirmaciones y su verificación. En el episodio de planeación se identificaron comportamientos relacionados con la elaboración de un plan y formas de proceder en atención a un objetivo definido, la escogencia o realización de acciones pertinentes, el cuestionamiento de alguna estrategia adoptada y su examen o análisis. El episodio de implementación dejó ver comportamientos correspondientes a la realización de acciones en consonancia con algún plan trazado, el control sobre su realización, el contraste entre las acciones realizadas y lo proyectado, el cuestionamiento de las acciones realizadas, la anticipación de resultados, el acceso a conocimiento relevante y el establecimiento de conclusiones apoyados en el trabajo realizado.

En el episodio de verificación se identificaron comportamientos como la representación de objetos o relaciones geométricas para verificar algún resultado, la revisión del trabajo realizado con el fin de no olvidar detalles, la revisión del enunciado del problema y lo que este solicita y la verificación de los resultados y la pertinencia de las respuestas. Finalmente, en el episodio de síntesis los comportamientos observados fueron el establecimiento de conclusiones apoyadas en la exploración realizada y la generalización de resultados a partir de la información recolectada.

\section{Análisis comparativo entre grupos}

En el análisis comparativo se tuvo en cuenta la naturaleza del proceso de resolución y la relación entre los episodios de resolución y los CM, ambos con la presencia de la GD. Presentamos una síntesis de estos asuntos.

\section{Naturaleza del proceso de resolución}

Sobre la frecuencia de los episodios se aprecia una alta presencia de acciones encaminadas a la planeación e implementación (figuras 7 y 8). Los episodios de implementación tienen frecuencia menor a los de planeación, lo que se sustenta en la naturaleza de estos dos episodios (tabla 1). Por otro lado, se reconoce una baja presencia de episodios de síntesis. Este episodio tuvo lugar cuando se daba fin a la exploración o descubrimiento de propiedades, formulando conjeturas que posteriormente se sometían a justificación. 


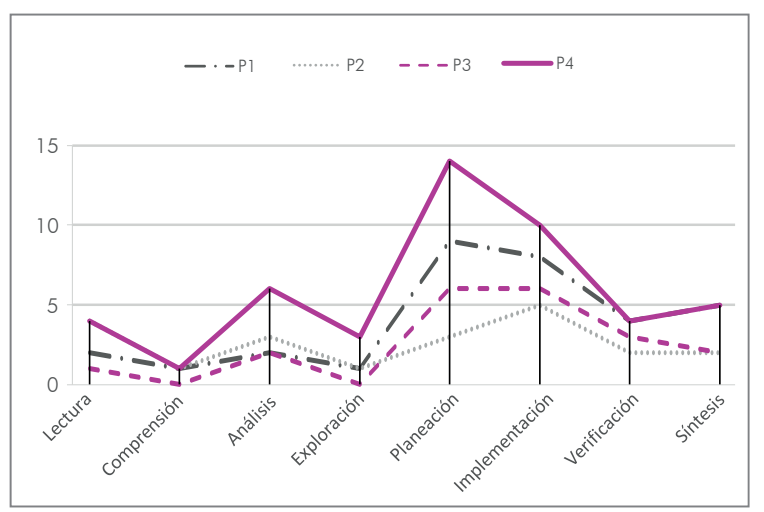

Figura 7. Frecuencia de episodios Caro y Paul. P1, P2, P3 y P4 son los problemas propuestos

Fuente: elaboración propia.

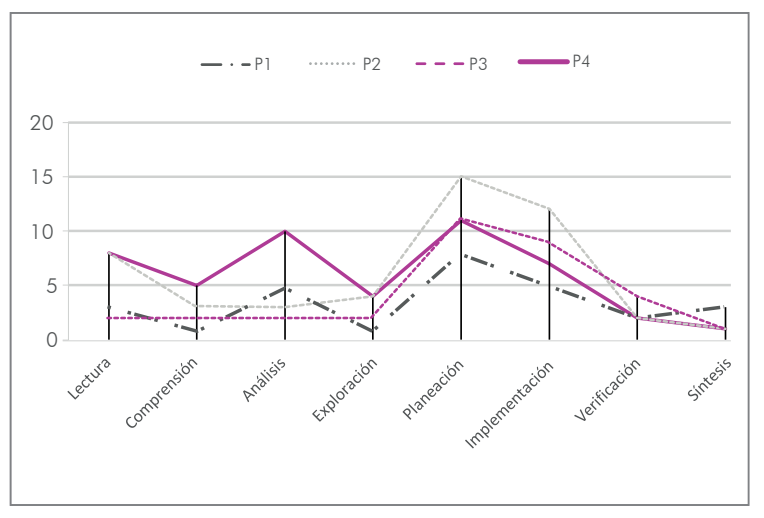

Figura 8. Frecuencia de episodios Ana y Juan. P1, P2, P3 y P4 son los problemas propuestos

Fuente: elaboración propia.

El episodio de verificación no reportó alta frecuencia. En ambos grupos se observó que este apareció cuando se ponía a prueba alguna hipótesis. Dada la naturaleza del ambiente computacional, las acciones de verificación estuvieron encaminadas a realizar construcciones robustas de los objetos geométricos. En estos diagramas se observa un comportamiento similar en las frecuencias de cada episodio para los cuatro problemas. Sin embargo, algunos aspectos fueron distintos en el trabajo realizado por los grupos, principalmente en los cuatro primeros episodios presentados en estos diagramas. Caro y Paul se limitaron a una lectura inicial del problema y a pocas acciones dirigidas a la comprensión de este, distinto a lo realizado por Ana y Juan.
Ana y Juan exhibieron mayor cantidad de acciones enfocadas al análisis. Esto puede observarse en el tipo de trabajo realizado al abordar cada problema. Mientras Caro y Paul se enfocaban en verificar el cumplimiento de propiedades en casos particulares, Juan y Ana se enfocaron en descubrir características suficientes y necesarias de objetos geométricos para que estas propiedades se satisficieran. Esto incidió en las conjeturas elaboradas por cada grupo. Las conjeturas de Caro y Paul no gozaron de una generalidad en su contenido, mientras que Juan y Ana sí lograron esto.

Al mirar la distribución del tiempo empleado en la resolución se ve que Paul y Caro realizaron principalmente acciones de implementación, seguido de acciones de análisis (figura 9). Algo distinto aconteció con Juan y Ana, quienes dedicaron en algunos problemas una cantidad de tiempo mayor para el análisis del problema y una menor cantidad al implementar acciones (figura 10). Autores como Cai (1994) mencionan que cuando los estudiantes dedican mayor cantidad de tiempo en la planeación y análisis del problema abordado, con respecto a la ejecución de acciones, obtienen mejores resultados que aquellos estudiantes que dedican una gran cantidad de tiempo realizando acciones sobre las cuales no hay un análisis o planeación.

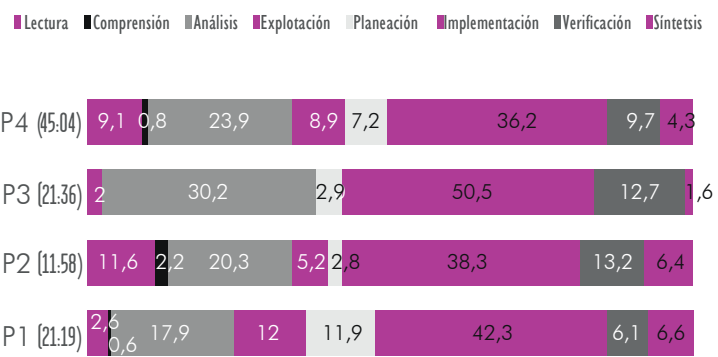

Figura 9. Porcentaje de duración episodios, grupo Caro y Paul

Fuente: elaboración propia. 


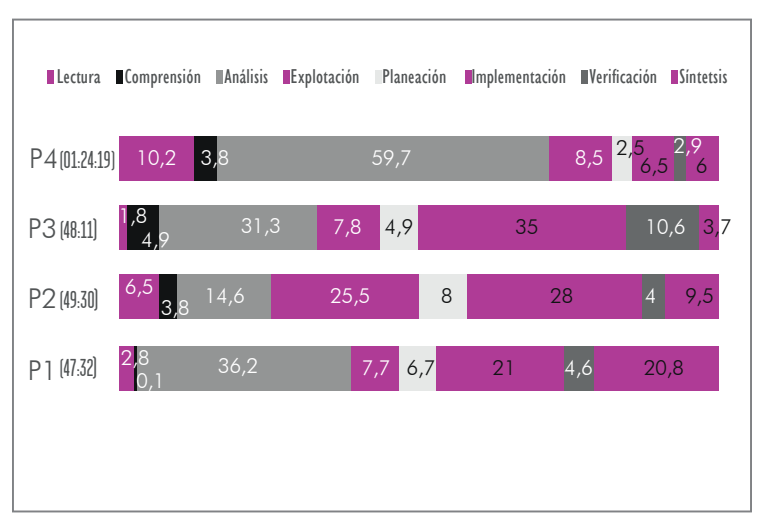

Figura 10. Porcentaje de duración episodios, grupo Ana y Juan

Fuente: elaboración propia.

\section{Un análisis de los CM}

Presentamos los CM observados en cada episodio del proceso de resolución. Queremos así reconocer diferencias y similitudes en el trabajo realizado y ver si la GD tuvo alguna incidencia. Una vez se identificaron los CM al resolver cada problema, se reconocieron cuáles eran más recurrentes en cada grupo. Para ello se realizó una lista de los CM identificados y esta se codificó, como se presenta en la tabla 5.

Tabla 5. Codificación de comportamientos metacognitivos

\begin{tabular}{|c|c|c|c|}
\hline$C M$ & Cód & $C M$ & Cód \\
\hline $\begin{array}{l}\text { Identificar partes del } \\
\text { problema que pudieran } \\
\text { omitirse }\end{array}$ & CMI & $\begin{array}{l}\text { Buscar información } \\
\text { en representaciones } \\
\text { gráficas construidas }\end{array}$ & CM21 \\
\hline $\begin{array}{l}\text { Lectura de partes } \\
\text { principales del } \\
\text { enunciado }\end{array}$ & CM2 & $\begin{array}{l}\text { Cuestionar acciones } \\
\text { realizadas }\end{array}$ & cM22 \\
\hline $\begin{array}{l}\text { Identificar o retomar } \\
\text { condiciones expuestas } \\
\text { en el enunciado del } \\
\text { problema }\end{array}$ & CM3 & $\begin{array}{l}\text { Generalizar resultados } \\
\text { a partir del trabajo } \\
\text { realizado }\end{array}$ & cM23 \\
\hline $\begin{array}{l}\text { Extraer información } \\
\text { de representaciones } \\
\text { gráficas }\end{array}$ & CM4 & $\begin{array}{l}\text { Revisar el trabajo } \\
\text { realizado para no } \\
\text { olvidar detalles }\end{array}$ & CM24 \\
\hline $\begin{array}{l}\text { Establecer conclusiones } \\
\text { gracias a exploración } \\
\text { en GD }\end{array}$ & CM5 & $\begin{array}{l}\text { Revisar el enunciado } \\
\text { del problema y lo que } \\
\text { solicita }\end{array}$ & cM25 \\
\hline $\begin{array}{l}\text { Usar representaciones } \\
\text { gráficas/simbólicas para } \\
\text { soportar/comunicar } \\
\text { ideas }\end{array}$ & CM6 & $\begin{array}{l}\text { Verificar resultados } \\
\text { y pertinencia de } \\
\text { respuestas obtenidas }\end{array}$ & CM26 \\
\hline Reconocer dificultades & $\mathrm{CM} 7$ & $\begin{array}{l}\text { Elaborar justificaciones } \\
\text { para soportar/rechazar } \\
\text { ideas }\end{array}$ & cM27 \\
\hline
\end{tabular}




\begin{tabular}{|c|c|c|c|}
\hline$C M$ & Cód & $C M$ & Cód \\
\hline $\begin{array}{l}\text { Acceder a conocimiento } \\
\text { relevante }\end{array}$ & CM8 & $\begin{array}{l}\text { Sintetizar o parafrasear } \\
\text { lo que el problema } \\
\text { solicita o presenta }\end{array}$ & CM28 \\
\hline $\begin{array}{l}\text { Manipular objetos } \\
\text { geométricos }\end{array}$ & CM9 & $\begin{array}{l}\text { Revisar el enunciado } \\
\text { del problema para } \\
\text { identificar partes del } \\
\text { problema }\end{array}$ & CM29 \\
\hline $\begin{array}{l}\text { Examinar posibles } \\
\text { relaciones entre objetos } \\
\text { geométricos construidos }\end{array}$ & CM10 & $\begin{array}{l}\text { Cuestionar el } \\
\text { conocimiento } \\
\text { involucrado o } \\
\text { afirmaciones realizadas }\end{array}$ & CM30 \\
\hline $\begin{array}{l}\text { Anticipar relaciones } \\
\text { geométricas }\end{array}$ & CMI 1 & $\begin{array}{l}\text { Reformular resultados/ } \\
\text { conclusiones para } \\
\text { que se ajuste a lo } \\
\text { descubierto }\end{array}$ & CM3 1 \\
\hline $\begin{array}{l}\text { Usar representaciones } \\
\text { gráficas para formular/ } \\
\text { reconocer resultados }\end{array}$ & CM12 & $\begin{array}{l}\text { Reconstruir argumentos } \\
\text { previamente } \\
\text { presentados }\end{array}$ & cM32 \\
\hline $\begin{array}{l}\text { Verificar la validez } \\
\text { o generalidad de } \\
\text { propiedades/relaciones }\end{array}$ & CM13 & $\begin{array}{l}\text { Indagar los motivos que } \\
\text { llevan al cumplimiento } \\
\text { de una propiedad }\end{array}$ & cM33 \\
\hline $\begin{array}{l}\text { Elaborar/modificar un } \\
\text { plan y proyectar formas } \\
\text { de proceder, dado un } \\
\text { objetivo }\end{array}$ & CMI 4 & $\begin{array}{l}\text { Sugerir nuevas acciones } \\
\text { para lograr objetivos o } \\
\text { metas trazadas }\end{array}$ & CM34 \\
\hline $\begin{array}{l}\text { Escoger/realizar } \\
\text { acciones pertinentes }\end{array}$ & CM15 & $\begin{array}{l}\text { Clarificar el objetivo del } \\
\text { problema o información } \\
\text { suministrada }\end{array}$ & cM35 \\
\hline $\begin{array}{l}\text { Cuestionar, analizar } \\
\text { o examinar alguna } \\
\text { estrategia adoptada }\end{array}$ & CMI 6 & $\begin{array}{l}\text { Evocar el objetivo el } \\
\text { problema }\end{array}$ & cM36 \\
\hline $\begin{array}{l}\text { Verificar los pasos } \\
\text { realizados y detalles del } \\
\text { plan trazado }\end{array}$ & CMl7 & $\begin{array}{l}\text { Dividir un problema en } \\
\text { otros más pequeños }\end{array}$ & CM37 \\
\hline $\begin{array}{l}\text { Realizar acciones en } \\
\text { consonancia con algún } \\
\text { plan trazado }\end{array}$ & CM18 & $\begin{array}{l}\text { Clarificar el objetivo de } \\
\text { un plan }\end{array}$ & cM38 \\
\hline $\begin{array}{l}\text { Controlar la realización } \\
\text { de acciones con base } \\
\text { en el plan trazado }\end{array}$ & CM19 & $\begin{array}{l}\text { Revisar las } \\
\text { construcciones } \\
\text { realizadas }\end{array}$ & CM39 \\
\hline $\begin{array}{l}\text { Contrastar las acciones } \\
\text { realizadas y lo } \\
\text { proyectado }\end{array}$ & CM20 & $\begin{array}{l}\text { Proponer distintas } \\
\text { formas de justificar una } \\
\text { idea }\end{array}$ & CM40 \\
\hline
\end{tabular}

Fuente: elaboración propia. 


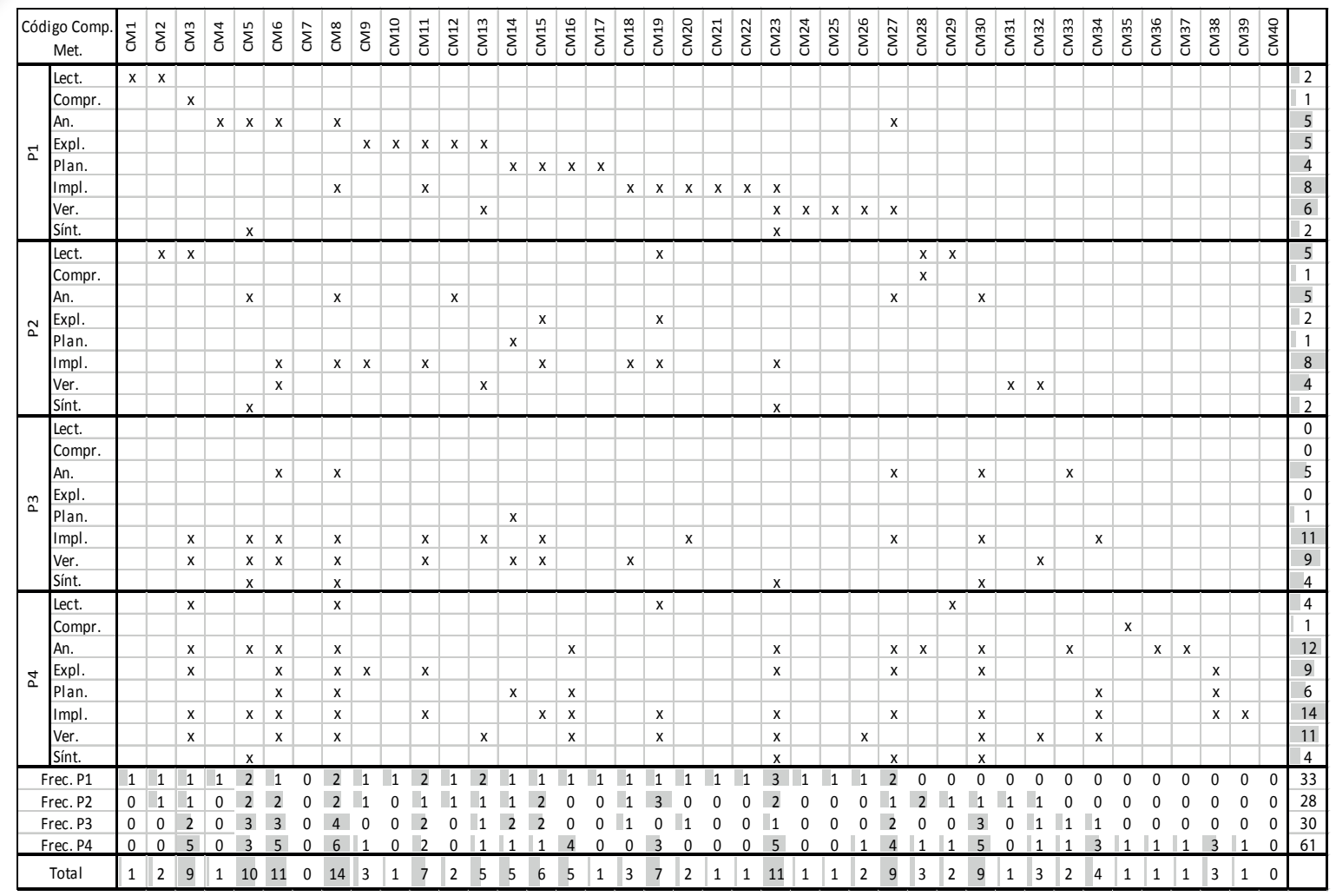

Figura 11. Comportamientos exhibidos por Caro y Paul

Fuente: elaboración propia.

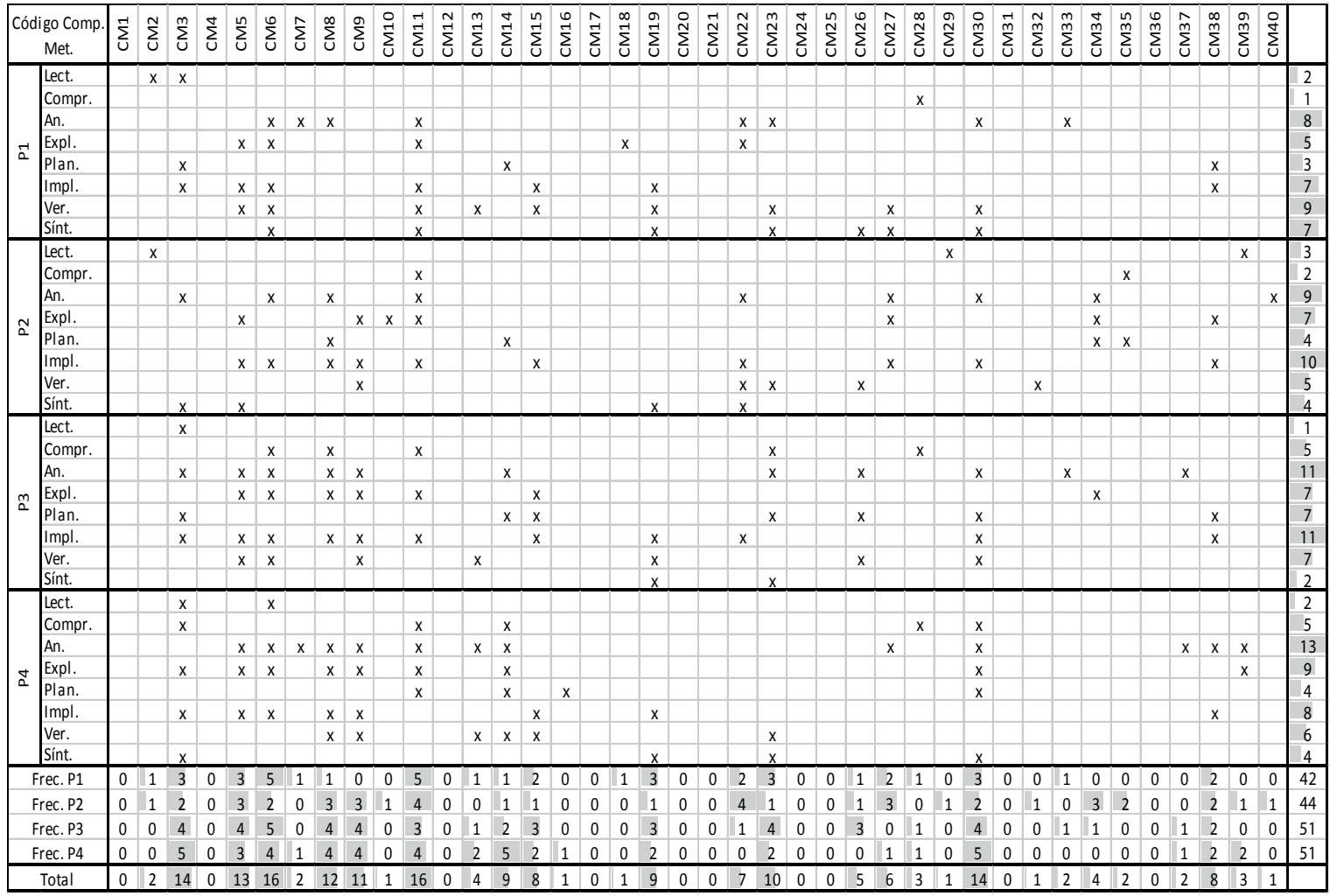

Figura 12. Comportamientos exhibidos por Ana y Juan

Fuente: elaboración propia. 
Posteriormente se elaboró un formato de doble entrada (figuras 11 y 12) en el que se colocaban los CM (columnas) y los episodios de resolución de cada problema (filas). Se realizaron marcas en los casos que un $\mathrm{CM}$ tenía presencia en algún episodio. Esto permitió reconocer los episodios que reportaban mayor cantidad o diversidad de CM, así como los comportamientos con mayor presencia (última fila).

Con esta información se analizó la presencia de CM en cada problema propuesto teniendo en cuenta los episodios de resolución. Dicho análisis dejó ver (figuras 13 y 14) aspectos similares en ambos grupos como lo es la poca presencia de CM en episodios de lectura o comprensión, lo que puede deberse a que estos episodios no presentaron una duración y frecuencia mayor, o la alta presencia de CM en episodios de implementación y verificación. Se observa que Ana y Juan desplegaron mayor cantidad de CM en los episodios de análisis, exploración y planeación, lo que significa que manifestaron mayor cantidad de acciones dirigidas al control, la regulación y el monitoreo al analizar el problema, planear acciones y explorar la situación propuesta con ayuda del software. Lo anterior aporta en la comprensión de la diferencia de los resultados obtenidos por cada pareja al resolver los problemas.

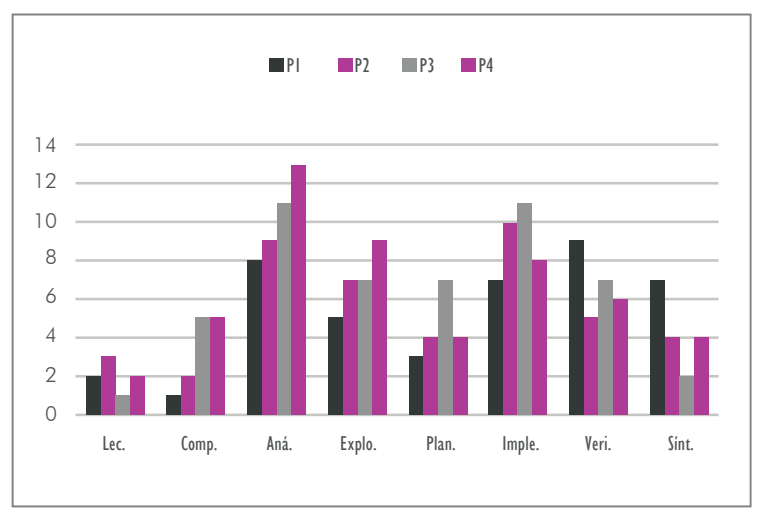

Figura 13. Frecuencia CM, grupo Ana y Juan Fuente: elaboración propia.

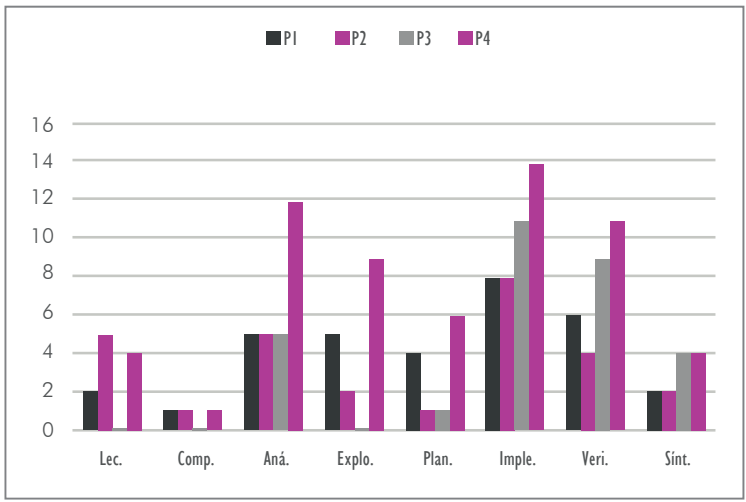

Figura 14. Frecuencia CM, grupo Caro y Paul

Fuente: elaboración propia.

Kim et al. (2013) resaltan el impacto de los recursos externos en los CM a nivel individual y social. En el trabajo realizado por los grupos con GD esto se ilustra, pues este realimentaba las acciones que los estudiantes realizaban y promovió CM no originados dentro de la interacción social. Al comparar la forma de involucrar GeoGebra, se reconocen dos esquemas de trabajo diferenciados que llevaron a resultados distintos en términos de la generalidad de las conjeturas. Mientras que Caro y Paul realizaron un trabajo enfocado en casos particulares, lo que permitió tener seguridad sobre el resultado obtenido dada la precisión del software, Ana y Juan utilizaron la función de arrastre, descubriendo propiedades que no caracterizaban a un tipo particular de cuadrilátero, sino a una familia de estos. El enfoque de Caro y Paul los llevó a obtener resultados particulares, dejando de lado un trabajo en el que la exploración favoreciera resultados más generales.

\section{Conclusiones}

Con el estudio se quería identificar la naturaleza del proceso de resolución de problemas de demostración, en los que tiene presencia la GD. Adicionalmente, se quería mostrar que los CM presentes en la resolución de estos 
problemas son un elemento que puede llevar a un grupo, con un conocimiento matemático reducido, a obtener mejores resultados que un grupo con mayor conocimiento de la disciplina. Para ello se tomaron dos grupos de futuros profesores de matemáticas con un nivel de formación distinta. Lo presentado en este documento aporta elementos al campo investigativo, pero no permite generalizar los resultados encontrados. Se requieren estudios en otros contextos que permitan avanzar en esta vía.

Respecto a los episodios de resolución, se reconoce la presencia de todos ellos en cada problema abordado, aunque su frecuencia no fuera similar, inclusive, se observó en ambos grupos un patrón asociado a la forma en que estos se manifestaron en cada problema. Mientras que episodios de implementación y planeación tuvieron gran presencia, episodios como verificación, comprensión y exploración mostraron una tendencia baja en general. Además, los episodios no se manifestaron en un orden lineal, lo que soporta la idea de Erbas y Okur (2012)problem solving episodes, and metacognitions of five Turkish high school freshmen and explore the interplay of these on their problem solving success in mathematics. Participants worked on ten problems selected among the released mathematical literacy items used in Programme for International Student Assessment (PISA y Kuzle (2015), quien resaltó esto al analizar el modelo propuesto por Polya. La configuración grupal y el acceso a la GD favoreció que cada episodio, y en consecuencia el proceso de resolución se viera permeado por CM. La interacción de los estudiantes y la realimentación ejercida por el software llevó a que ellos ampliaran sus miradas y ajustaran sus acciones, aspecto que difícilmente se lograría en solitario o en lápiz y papel. Esto deja ver, en la misma vía que Furinghetti y Morselli (2009), que el conocimiento, aspecto de orden cognitivo, no es suficiente para dar respuesta adecuada a un problema.

Con relación al trabajo grupal, los estilos de trabajo, aun cuando diferentes, permitieron reconocer CM característicos de cada grupo e inclusive algunos compartidos entre ellos. La presencia de CM comunes podría sustentarse en la metodología involucrada en los espacios académicos de geometría que ellos cursaron. Pareciera que la aproximación metodológica propuesta por el grupo $A E \bullet G$ (Perry et al., 2013) promueve, a través de la gestión del profesor, el desarrollo de CM en los estudiantes desde niveles tempranos de formación, los cuales son de utilidad al afrontar problemas de demostración. La metodología de estos espacios formativos pertenecería así al conjunto de enfoques instruccionales que promueven habilidades metacognitivas (Kim et al., 2013), las cuales, como se ha documentado, pueden ser transferidas a otros contextos o problemas abordados.

Respecto al recurso externo, la GD fue relevante en la resolución de problemas. Su naturaleza provocó que los estudiantes reconocieran en este recurso una herramienta de validación y soporte para las acciones ejecutadas y resultados 
obtenidos. Este recurso promueve CM como la planeación de estrategias y su implementación. También, el software permite que comportamientos dirigidos al control de las acciones realizadas y la verificación de resultados se puedan llevar a cabo, estableciendo resultados cuyo soporte puede ser netamente empírico o involucrar elementos teóricos. Lo anterior brinda evidencia sobre la forma en que la GD apoya la metacognición en un nivel individual y grupal, pues realimenta las acciones de los estudiantes, modifica sus concepciones y amplía su visión y comprensión.

El cuadro comparativo realizado, que involucraba parejas con formación matemática y experiencias en el desarrollo de problemas de demostración distinta, dejó ver que aquellos estudiantes que gozaban de profundidad en su formación académica, reconocimiento y uso de la GD y antecedentes en los que habían encarado problemas de demostración con ayuda de este recurso computacional, no reportó mejores resultados que el grupo de estudiantes que apenas había cursado un primer espacio académico de geometría. Las diferencias mostradas frente a la presencia y duración de los episodios de resolución, así como la diversidad y frecuencia de CM en estos, a favor de Ana y Juan, permiten comprender estos resultados. Sin embargo, aun cuando estos resultados se apoyan en estilos de trabajo diferenciados, no tenemos evidencia para asegurar que estos estilos sean comunes entre estudiantes con una formación académica similar. Es posible que estudiantes con una formación matemática reducida realicen un trabajo apoyado en casos particulares, como el realizado por Caro y Paul.

\section{Referencias}

Baxter, P. y Jack, S. (2008). Qualitative case study methodology: Study design and implementation for novice researchers. The Qualitative Report, 13(4), 544-559. https://doi.org/ http://dx.doi.org/9771682584003-32963

Cai, J. (1994). A protocol-analytic study of metacognition in mathematical problem solving. Mathematics Education Research Journal, 6(2), 166-183. https://doi.org/10.1007/ BF03217270

Chiu, M. M., Jones, K. A. y Jones, J. L. (2013). Building on Schoenfeld's studies of metacognitive control towards social metacognitive control. En Y. Li y J. Moschkovich (eds.), Proficiency and beliefs in learning and teaching mathematics: Learning from Alan Schoenfeld and Günter Törner (pp. 69-85). Róterdam: Sense Publishers. https://doi. org/10.1007/978-94-6209-299-0

Erbas, A. K. y Okur, S. (2012). Researching students' strategies, episodes, and metacognitions in mathematical problem solving. Quality and Quantity, 46(1), 89-102. https:// doi.org/10.1007/s $11135-010-9329-5$

Furinghetti, F. y Morselli, F. (2009). Every unsuccessful problem solver is unsuccessful in his or her own way: Affective and cognitive factors in proving. Educational Studies in Mathematics, 70(1), 71-90. https://doi. org/10.1007/s10649-008-9134-4

Hanna, G. (2000). Proof, explanation and exploration: An overview. Educational Studies in Mathematics, 44(1-3), 5-23. https://doi. org/10.1023/A:1012737223465

Karsli, T. A. (2015). Relation among meta-cogntition level, decision making, problem solving and locus of control in a Turkish adolescent population. Procedia-Social and Behavioral Sciences, 205, 35-42. https://doi. org/10.1016/j.sbspro.2015.09.008 
Kim, Y. R., Park, M. S., Moore, T. J. y Varma, S. (2013). Multiple levels of metacognition and their elicitation through complex problem-solving tasks. Journal of Mathematical Behavior, 32(3), 377-396. https://doi.org/10.1016/i.jmathb.2013.04.002

Koichu, B. y Leron, U. (2015). Proving as problem solving: The role of cognitive decoupling. Journal of Mathematical Behavior, 40, 233-244. https://doi.org/10.1016/j.jmathb.2015.10.005

Kuzle, A. (2015). Nature of metacognition in a dynamic geometry environment. Lumat, 3(5), 627-646.

Lin, X. y Sullivan, F. R. (2008). Computer contexts for supporting metacognitive learning. En J. Voogot y G. Knezek (eds.), International handbook of information technology in primary and secondary education (pp. 281-298). Boston: Springer. https://doi.org/10.1007/978-0-387-73315-9_17

Marrades, R. y Gutiérrez, Á. (2000). Proofs produced by secondary school students learning geometry in a dynamic computer environment. Educational Studies in Mathematics, 44(1-3), 87-125. https://doi.org/10.1023/A:1012785106627

Nunokawa, K. (2010). Proof, mathematical problem-solving, and explanation in mathematics teaching. En G. Hanna, H. N. Jahnke y H. Pulte (eds.), Explanation and proof in mathematics: philosophical and educational perspectives (pp. 223236). Boston: Springer. https://doi.org/10.1007/978-1-4419-0576-5_15

Perry, P., Samper, C., Camargo, L. y Molina, O. (2013). Innovación en un aula de geometría de nivel universitario. En Geometría plana: un espacio de aprendizaje (pp. 11 -34). Bogotá: Fondo Editorial Universidad Pedagógica Nacional.

Raes, A., Schellens, T., De Wever, B. y Benoit, D. F. (2016). Promoting metacognitive regulation through collaborative problem solving on the web: When scripting does not work. Computers in Human Behavior, 58, 325-342. https://doi.org/10.1016/j.chb.2015.12.064

Schneider, W. y Artelt, C. (2010). Metacognition and mathematics education. zdmInternational Journal on Mathematics Education, 42(2), 149-161. https://doi. org/10.1007/s 1 1858-010-0240-2

\section{Para citar este artículo}

Sua, C. (2019). Saber suficiente no es suficiente: comportamientos metacognitivos al resolver problemas de demostración con el apoyo de la geometría dinámica. Tecné, Episteme y Didaxis: TED, 45, 121-142. 